\title{
Evaluation of Biotinylated PAMAM Dendrimer Toxicity in Models of the Blood Brain Barrier: A Biophysical and Cellular Approach
}

\author{
Heather A. Bullen ${ }^{1}$, Ruth Hemmer ${ }^{2}$, Anthony Haskamp ${ }^{1}$, Chevelle Cason ${ }^{1}$, Stephen Wall, \\ Robert Spaulding ${ }^{2}$, Brett Rossow ${ }^{2}$, Michael Hester ${ }^{2}$, Megan Caroway ${ }^{2}$, Kristi L. Haik ${ }^{2}$ \\ ${ }^{1}$ Department of Chemistry, Northern Kentucky University, Highland Heights, USA; ${ }^{2}$ Department of Biological Sciences, Northern \\ Kentucky University, Highland Heights, USA. \\ E-mail: \{bullenh1, haikk\}@nku.edu
}

Received October $12^{\text {th }}, 2011$; revised November $17^{\text {th }}, 2011$; accepted November $26^{\text {th }}, 2011$.

\begin{abstract}
The interaction of biotinylated G4 poly (amidoamine) (PAMAM) dendrimer conjugates and G4 PAMAM dendrimers with in vitro models of the blood brain barrier (BBB) was evaluated using Langmuir Blodgett monolayer techniques, atomic force microscopy (AFM) and lactate dehydrogenase measures of cell membrane toxicity. Results indicate that both G4 and G4 biotinylated PAMAM dendrimers disrupt the composition of the liquid condensed (LC) and liquid expanded (LE) phases of the 1,2-dipalmitoyl-sn-glycero-3-phosphocholine (DPPC) lipid monolayer. The disruption is concentration dependent and more marked for G4 biotinylated PAMAMs. Lactate dehydrogenase (LDH) assays using endothelial cell culture models of the BBB indicate that biotinylation results in higher levels of toxicity than non-biotinylation. This approach provides valuable information to assess nanoparticle toxicity for drug delivery to the brain.
\end{abstract}

Keywords: Dendrimers, Drug Delivery, Blood Brain Barrier, Toxicity

\section{Introduction}

Central nervous system (CNS) diseases, disorders and injuries affect over 1.5 billion people worldwide and diagnosis and treatment of CNS disorders represent a considerable challenge. The brain is separated from the rest of the body by the blood brain barrier (BBB) and the protective mechanisms of the $\mathrm{BBB}$ renders the brain a site of poor permeability of various drugs and delivery systems. The BBB is a tight seal of cells that lines the blood vessels in the brain. The walls of BBB capillaries are composed of specialized endothelial cells, brain capillary endothelial cells (BCEC), which form tight junctions. Tight junctions contain integral membrane proteins that form a seal between adjacent endothelial cells. In addition, accessory structures that surround the BCECs include pericytes and associated astrocytes and neurons [1-3]. The complexity of the BBB comes from the variety and number of transporters within the BBB, including p-glycoprotein-1, glucose-related transporters, nucleoside transporters, receptors for transferrin, insulin, leptin, lectins, IGFs, various ATPases and many, many more that are taken up via receptor mediated endocytosis [4].
This complexity also makes developing in vitro models of the BBB challenging.

While the BBB is essential for maintaining a healthy brain, it impedes efforts to deliver therapeutic agents into the brain. The poor permeability of various drugs as well as delivery systems across the BBB is primarily due to tight junctions, lack of capillary fenestrations and presence of efflux transporters. The BBB can reportedly block more than $98 \%$ of CNS drugs [5]. Due to the ineffectiveness of conventional drug therapies, finding ways to deliver therapeutic drugs to the CNS safely and effectively is essential. The development of novel strategies that could overcome the obstacles of brain drug delivery is essential. The application of nanoscience to CNS disorders is an active area of research. A number of nanoparticle delivery systems have been developed and demonstrated promising properties [5-8].

Dendrimers are appealing choices for nanoparticle drug delivery because of the ability to control their precise architecture, size and shape, high uniformity and purity, high loading capacity, low toxicity and low immunogenicity [9-12]. The presence of a large number of 
surface groups provides opportunity to conjugate ligands not only for transport across the BBB but also for targeting specific cells, such as tumors. Dendrimers can be prepared with specific surface modifications that enable the dendrimers to gain entry through a membrane while holding a molecule that cannot pass on its own. Once the dendrimer passes the membrane, it can deliver the drug held in its interior.

There are a tremendous number of potential applications for dendrimers in biological systems [13-18], with poly(amidoamine) PAMAM dendrimers being the most widely studied [11] However, the application of dendrimers in brain delivery is a relatively new area of research. Several targeted drug delivery systems using various targeting ligands have been used with some success in terms of BBB crossing [5,8], including lactoferrin [19], epidermal growth factors [20] and doxorubicin [21]. The mechanism of uptake and toxicity to the BBB has not been extensively studied. A detailed characterization of dendrimer toxicity is important for the design and use of dendrimers in brain drug delivery. Toxicity of both the functional group and generation of the dendrimer must be taken into consideration. PAMAM dendrimers have been shown to be haemolytic and cytotoxic, with toxicity tending to be higher for cationic PAMAM dendrimers and to increase with generation $[22,23]$.

In this study we evaluated the potential toxicity of biotinylated G4 PAMAM dendrimer conjugates. Biotin is an important molecule used in several metabolic pathways and belongs to a family of molecules that have been shown to cross the BBB [24,25]. Biotin-labeled dendrimers have been utilized in tumor [26] and antibody [27] targeting studies and biosensor design [28]. Biotinylated PAMAM dendrimers may also have the potential for delivering therapeutic drugs to the brain $[24,29]$.

The biophysical interactions of biotinylated G4 PAMAM conjugates and G4 PAMAMs with lipid model membranes were evaluated using Langmuir Blodgett monolayer techniques and atomic force microscopy (AFM). Results were correlated with cellular toxicity measurements using endothelial cell culture models of the BBB. This work reports the first analysis of PAMAM dendrimers using this combined approach. The results provide important insights into strategies for developing nanoparticle systems for brain drug delivery.

\section{Experimental Section}

\subsection{Materials}

Poly(amidoamine) PAMAM dendrimers [core: ethylene diamine]; $(\mathrm{G}=4)$; dendri-PAMAM- $\left.\left(\mathrm{NH}_{2}\right)_{32}\right)$ were obtained from Dendritic Nanotechnologies, Inc. (Mt. Plea- sant, MI). Biotinylated PAMAMs were prepared using sulfo-NHS-LC-biotin (Pierce EZ-Link ${ }^{\circledR}$ Kit) as described previously [30]. Biotinylated dendrimers were resuspended $(1.0 \mathrm{mg} / \mathrm{mL})$ in $1.0 \mathrm{M}$ phosphate buffer saline (PBS) until used.

1,2-dipalmitoyl-sn-glycero-3-phosphocholine (DPPC) was obtained from Avanti Lipids (Alabaster, AL). All organic solvents used were analytical, HPLC grade, from Sigma (Sigma-Aldrich, St. Louis, MO). DI water was obtained using a Milli-Q plus water purification system (Millipore, Bedford, MA, USA). PBS and Borate buffers were prepared from Pierce buffer packs (Pierce Protein Research Products; Rockford, IL).

\subsection{Langmuir Trough}

A KSV 2000 Langmuir-Blodgett trough (KSV, Helsinki) was used for lipid monolayer experiments. Interfacial pressures were measured with a Wilhelmy balance using a platinum plate and an aqueous subphase, DI water, was used for all experiments. Before each experiment the trough was cleaned twice with 200-proof ethanol and DI water. All impurities remaining on surface were removed by aspirating the subphase surface. To ensure the trough was clean, the stability of the surface potential was checked before each experiment. Lipid and dendrimer solutions were prepared $(0.5 \mathrm{mg} / \mathrm{mL})$ in HPLC grade chloroform immediately prior to use. Lipid/dendrimer solutions were prepared by mixing dendrimers with the DPPC solution $(0.5 \mathrm{mg} / \mathrm{mL})$ corresponding to various lipid/dendrimer ratios (v/v). Monolayers were spread (60 $\mu \mathrm{L}$ of lipid or $60 \mu \mathrm{L}$ lipid/dendrimer mixtures) onto clean subphase $\left(25^{\circ} \mathrm{C} \pm 1{ }^{\circ} \mathrm{C}\right)$ using a Hamilton syringe. After evaporation of solvent $(20 \mathrm{~min})$ they were compressed as a rate of $5.5 \mathrm{~mm} / \mathrm{min}$. For monolayer transfer experiments a cleaved mica substrate was submerged into the subphase prior to addition of lipids. The lipid monolayer was spread as before and compressed to a desired surface pressure. The mica was then withdrawn from the subphase vertically a pulling rate of $5.5 \mathrm{~mm} / \mathrm{min}$ once target surface pressure had been reached.

\subsection{AFM Analysis of Monolayers}

A Dimension 3100 Digital Instruments SPM was utilized in tapping mode (NSC 14 tip-140 Hz) for all image analysis. Typical scan rates were $1 \mathrm{~Hz}$ and all images were acquired in air. Lipid monolayers were prepared by Langmuir Blodgett techniques, described above and were prepared on freshly cleaved mica substrates. Samples were stored in sealed containers and imaged immediately after film deposition. Multiple analyses of transferred monolayers was conducted. 


\section{4. bEnd.3 Cell Culture}

A murine brain capillary endothelial cell line, bEnd.3, was purchased from American Type Culture Collection (ATCC, CRL-2299) and cultured as recommended by the supplier. Briefly, bEnd.3 cells were cultured in media containing Dulbecco's Modified Eagle's Medium (DMEM; ATCC, 30 - 2002) containing $4 \mathrm{mM}$ L-glutamine, 4500 $\mathrm{mg} / \mathrm{L}$ glucose, $1 \mathrm{mM}$ sodium pyruvate, $1500 \mathrm{mg} / \mathrm{L}$ sodium bicarbonate and supplemented with penicillin (500 $\mathrm{u} / \mathrm{mL})$, streptomycin $(100 \mu \mathrm{g} / \mathrm{mL})$ and $10 \%$ fetal bovine serum (FBS) in a humidified $5 \% \mathrm{CO}_{2}, 95 \%$ air incubator at $37^{\circ} \mathrm{C}$. At $80 \%$ confluency, cells were trypsinized, counted and seeded in 96-well flat-bottomed plates with a density of $1.7 \times 10^{5}$ cells/well. After the cells grew for 2 days, cells were rinsed once with media and cells were then treated with one of the following treatment groups: $3 \mu \mathrm{L}$ of $1 \mathrm{mg} / \mathrm{mL} \mathrm{G} 4$ biotinylated dendrimers; $3 \mu \mathrm{L}$ of 1 $\mathrm{mg} / \mathrm{mL} \mathrm{G4}$ dendrimers; $3 \mu \mathrm{L}$ of culture media alone; or 3 $\mu \mathrm{L}$ of $0.05 \%$ sodium azide (each added to $197 \mu \mathrm{L}$ of media). After $24 \mathrm{~h}$ of treatment (in humidified $5 \% \mathrm{CO}_{2}$, $95 \%$ air incubator at $37^{\circ} \mathrm{C}$ ) the media was collected and used for LDH analysis. Fresh media was added to wells and after $24 \mathrm{~h}$ media was collected again for LDH analysis. Cell phenotype was confirmed by an immunocytochemical stain for von Willebrand factor [31].

\subsection{Lactate Dehydrogenase Assay (LDH)}

Mouse cerebral endothelial cell death was quantitatively assessed using the Tox-7 LDH based in vitro toxicology assay kit (Sigma). An LDH assay was performed on media collected after 24 and $48 \mathrm{~h}$. LDH values were compared between control and treatment conditions using a one-way ANOVA (SPSS) and Bonferroni post-hoc analyses when appropriate.

\section{Results and Discussion}

\subsection{Compression Isotherms}

Compression isotherms provide information on if PAMAM dendrimers penetrate into the lipid monolayer and influence lipid organization. The surface pressurearea isotherms ( $\pi$-A isotherms) for DPPC and DPPC with G4 PAMAM and G4 biotinylated PAMAM dendrimers are shown in Figure 1. The isotherm for pure DPPC is similar to that reported in the literature [32,33] and shows the well-known phase transition from liquid-expanded (LE) to liquid condensed (LC) phases at around $10 \mathrm{mN} / \mathrm{m}$. Addition of G4 and G4 biotinylated PAMAM dendrimers leads to a slight expansion in the disordered LE phase of the DPPC monolayer and the formation of an indistinct phase transition region. Increasing amounts of G4 and G4 biotinylated PAMAM dendrimers seem to have a greater effect on the disappearance of the DPPC LE-LC phase transition. The PAMAM dendrimers also affected the collapse pressure of the DPPC monolayer $(55 \mathrm{mN} / \mathrm{m})$; in general, higher concentrations of PAMAM dendrimers lead to a lower collapse surface pressure. This disruption of lipid monolayer stability is more pronounced for G4 biotinylated PAMAM dendrimers (Figure 1(B)).

These results indicate that both G4 and G4 biotinylated PAMAM dendrimers are initially incorporated into the DPPC monolayer. This incorporation disturbs the organization of the DPPC monolayer, as evident by

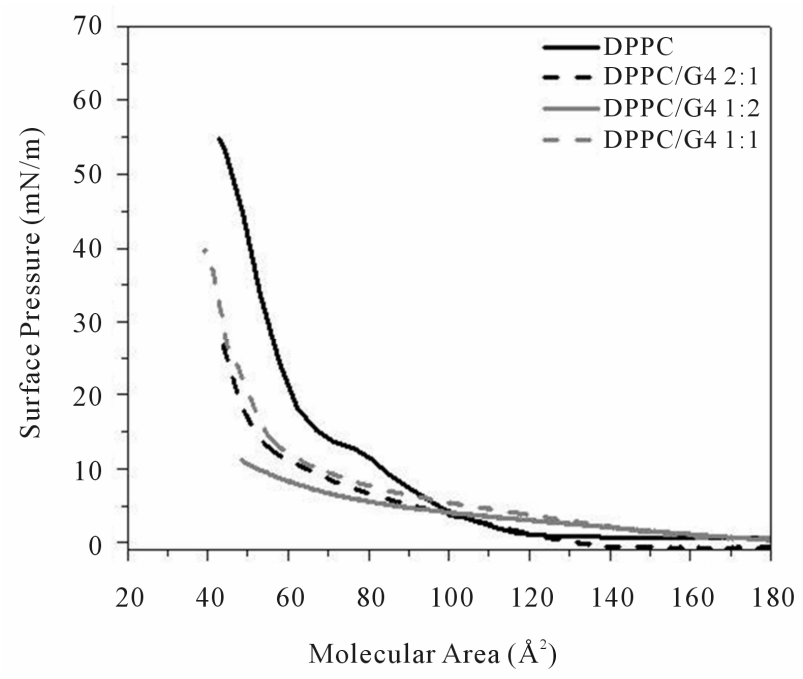

(A)

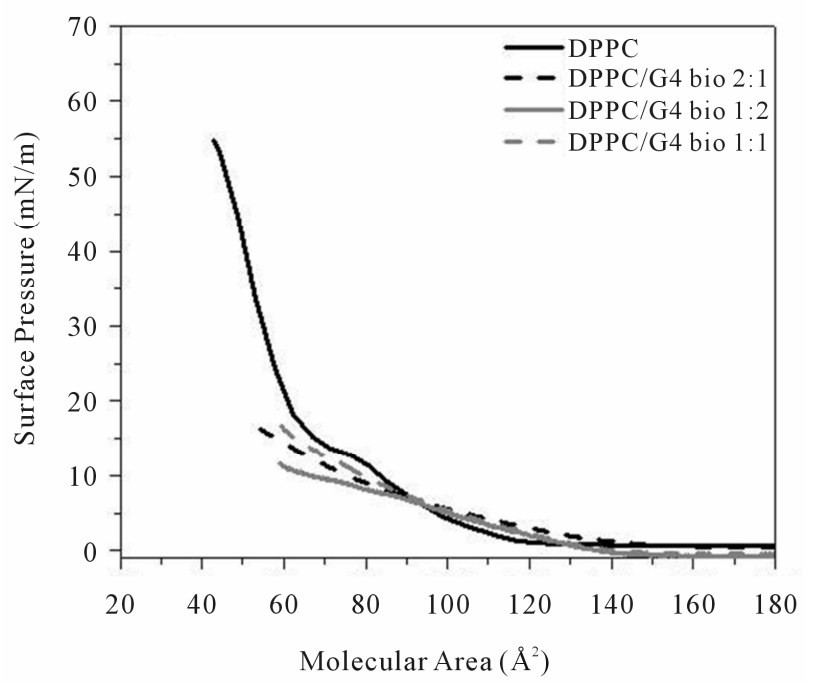

(B)

Figure 1. Surface pressure vs. area isotherm of DPPC lipid monolayers in the presence of different concentrations (v/v) of (A) G4 PAMAM dendrimers and (B) G4 biotinylated PAMAM dendrimers. 
the shift of the overall isotherm curve to lower molecular areas, loss of the LE-LC phase transition region and decrease of the surface collapse pressure. The PAMAM dendrimers fluidize the monolayer at low surface pressures, causing more lipid molecules to exist in the LE state rather than the LC phase [34-36]. Upon compression an enhanced squeeze out of material into the subphase is observed for both DPPC/G4 and DPPC/G4 biotinylated PAMAM dendrimer mixtures, as indicated by the shift of the isotherms to lower molecular areas and the kink evident at $\sim 6 \mathrm{mN} / \mathrm{m}[34,36]$. Previous research suggests that PAMAM dendrimers have an electrostatic nature of binding to lipid membranes [37], implying that primarily the polar head groups of DPPC and the surface amino groups of the G4 PAMAM dendrimers are involved in the interaction. This interaction would slightly affect the packing of the acyl chain domain, leading to the area expansion evident in the LE phase [38]. The comparable increase in area expansion in the LE region suggests that the G4 biotinylated PAMAM dendrimers affect the packing density of DPPC in a similar fashion.

\subsection{AFM Analysis}

AFM is a direct imaging tool for visualizing the lipid monolayers to determine the influence of G4 and G4 biotinylated PAMAM dendrimers on changes in the microdomain formation of the LE and LC phases. The AFM analysis of pure DPPC monolayer deposited at 12 $\mathrm{mN} / \mathrm{m}$ is shown in Figure 2. At this surface pressure, the LE and LC phases coexist, with the bright regions corresponding to the LC phase (a more upright configuration) and the dark regions corresponding to the LE phase. The LC phase domains are $\sim 2.2 \pm 0.6 \mu \mathrm{m}$ long and $\sim 124 \pm 35$ $\mathrm{nm}$ wide. These domains are separated by regions of the LE phase (gap is on average $\sim 200 \mathrm{~nm}$ ). Within the LE

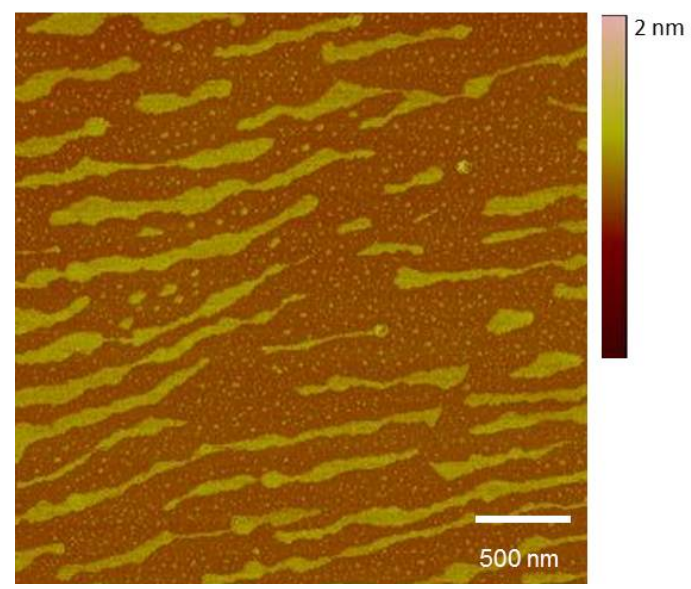

Figure 2. AFM height analysis of DPPC lipid monolayer (12 $\mathbf{m N} / \mathbf{m})$. phase region smaller islands of LC phase are also evident ( $\sim 30$ - $40 \mathrm{~nm}$ in diameter).

The influence of G4 and G4 biotinylated PAMAM dendrimers on the DPPC domain structure is shown in Figures 3 and 4. Increasing concentrations of G4 PAMAM dendrimers reduce the formation of the LC phase domains. At low G4 PAMAM dendrimer concentrations, DPPC:G4, 2:1 (Figure 3(A)), some regions look similar to DPPC alone, with long "stripe-like" LC phase domains $(\sim 1.0 \pm 0.2 \mu \mathrm{m}$ long; $\sim 68 \pm 29 \mathrm{~nm}$ wide) separated by $\sim 150-200 \mathrm{~nm}$ LE phase domains. However other regions show a more significant change in LC phase domain size, as evident by a more "oval, island-like" structures $(\sim 95 \pm 20 \mathrm{~nm}$ diameter) separated by $\sim 50 \mathrm{~nm}$ LE phase domains. Upon increasing concentration of G4 PAMAM dendrimers (DPPC:G4, 1:1), the "oval, island-like" LC phase regions become more prevalent within the mono- layer (Figure 3(B)). The LC phase islands ( $\sim 150 \pm 45 \mathrm{~nm}$ in diameter) are separated by $\sim 50$ $\mathrm{nm}$ LE phase domains. In addition a "honeycomb-like" LC phase structure is also evident, exhibiting larger regions of LE phase within the monolayer. Under higher concentrations of G4 PAMAM dendrimers (DPPC:G4, 1:2) two different domain structures are apparent, each leading to a further increase in the LE phase composition (Figures 3(C) and 3(D)). Some regions of the monolayer (Figure 3(C)) show LC phase islands that are quite varied in shape (roughly $\sim 500 \mathrm{~nm}$ diameter) separated by LE phase regions ( $\sim 300-400 \mathrm{~nm}$ gaps), which make up $\sim 68 \%$ of the monolayer composition. Other regions show further in- creases in LE phase composition (Figure 3(D)) with the LC phase existing as lines ( $720 \pm 180 \mathrm{~nm}$ long; $\sim 31 \pm 4 \mathrm{~nm}$ wide) separated by LE phase regions ( $\sim 300-$ $350 \mathrm{~nm}$ gaps), which make up $\sim 80 \%$ of the monolayer composition.

Low concentrations of G4 biotinylated PAMAM dendrimers, DPPC:G4bio, 2:1 (Figure 4(A)), cause an aggregation of the LC phase to form larger stripes compared to those found in DPPC alone. The LC and LE phase domains coexist as alternating stripes ( $>3 \mu \mathrm{m}$ long; $\sim 273 \pm 86 \mathrm{~nm}$ wide); within the LE regions, small islands $(\sim 35-50 \mathrm{~nm}$ diameter) of the LC phase also exist. Upon increasing concentration of G4 biotinylated PAMAM dendrimers (DPPC:G4bio, 1:1), two different domain structures are evident (Figures 4(B) and 4(C)), both showing an increase in the LE phase composition of the monolayer. Figure 4(B) shows LC phase stripe domains $(\sim 800 \pm 150 \mathrm{~nm}$ long, $\sim 210 \pm 80 \mathrm{~nm}$ wide) separated by LE phase regions ( $\sim 400 \mathrm{~nm}-1.5 \mu \mathrm{m}$ gaps), which make up $\sim 80 \%$ of the monolayer composition. Figure 4(C) shows LC islands domains that vary in shape (roughly 1 $\mu \mathrm{m}$ in diameter) separated by LE phase regions $(\sim 350 \mathrm{~nm}$ 

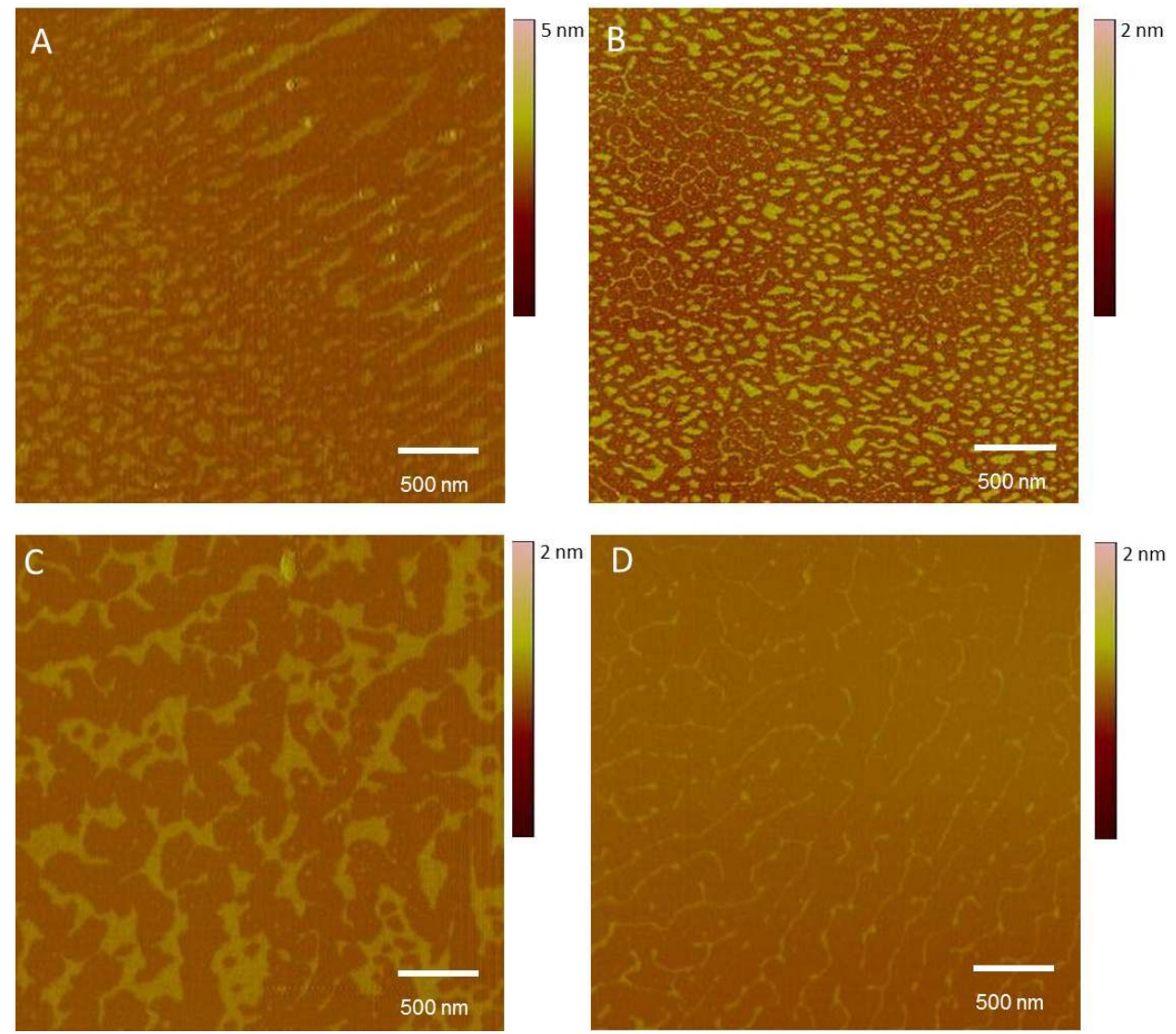

Figure 3. AFM height analysis of DPPC lipid monolayers $(12 \mathrm{mN} / \mathrm{m})$ in the presence of different concentrations (v/v) of G4 PAMAM dendrimers: (A) DPPC:G4, 2:1; (B) DPPC:G4, 1:1; (C) DPPC:G4, 1:2; (D) DPPC:G4, 1:2.

- $600 \mathrm{~nm}$ gaps), which makeup $\sim 65 \%$ of the monolayer composition. Under higher concentrations of G4 biotinylated PAMAM dendrimers (DPPC:G4bio, 1:2) a further increase in the LE phase region is evident (Figure 4D). The LC phase exists as lines $(\sim 716 \pm 210 \mathrm{~nm}$ long; $\sim 24 \pm 6 \mathrm{~nm}$ wide) separated by LE phase regions $(\sim 250$ $\mathrm{nm}$ gaps). Larger regions of LE phase $(1-2 \mu \mathrm{m})$ are also evident, with the LE phase making up $\sim 89 \%$ of the mono- layer composition.

AFM analysis reveals that both G4 and G4 biotinylated PAMAM dendrimer conjugates disrupt the composition of the LC and LE phases of DPPC. The disrupttion is concentration dependent and more marked for G4 biotinylated PAMAM dendrimers. These findings are in agreement with compression isotherm measurements, which showed a change in the LE-LC phase transition and decrease in collapse pressure, reflecting a clear destabilization of the DPPC packing upon addition of the PAMAM dendrimers. AFM analysis showed no evidence of PAMAM dendrimers within the monolayers. This is also in agreement with compression isotherm analysis which indicated that there was a squeeze out of material from the monolayer upon compression to higher surface pressures. The occupation of lower molecular areas for the isotherm suggests that in addition to the PAMAM dendrimers, lipids may also have been expelled from the monolayer. One possible mechanism could be that strong lipid-polymer interactions cause the DPPC molecules to surround the dendrimers, forming vesicles in solution that escape into the subphase [39].

\subsection{Cell Toxicity}

The bEnd.3 immortalized endothelial cell line was chosen as a basic model of the BBB. Endothelial cells make up a majority of the BBB, which controls cerebral homeostasis and prevents toxins and other chemicals in the blood stream from entering the brain. Therefore, it is vital to understand how nanoparticles affect these cells. The endothelial phenotype of the bEnd.3 cell line was confirmed by the observed expression of von Willebrand factor and uptake of fluorescently labeled low density lipoprotein (LDL) [31].

To obtain a specific measure of toxicity, the LDH assay was used for spectrophotometric measurement of 

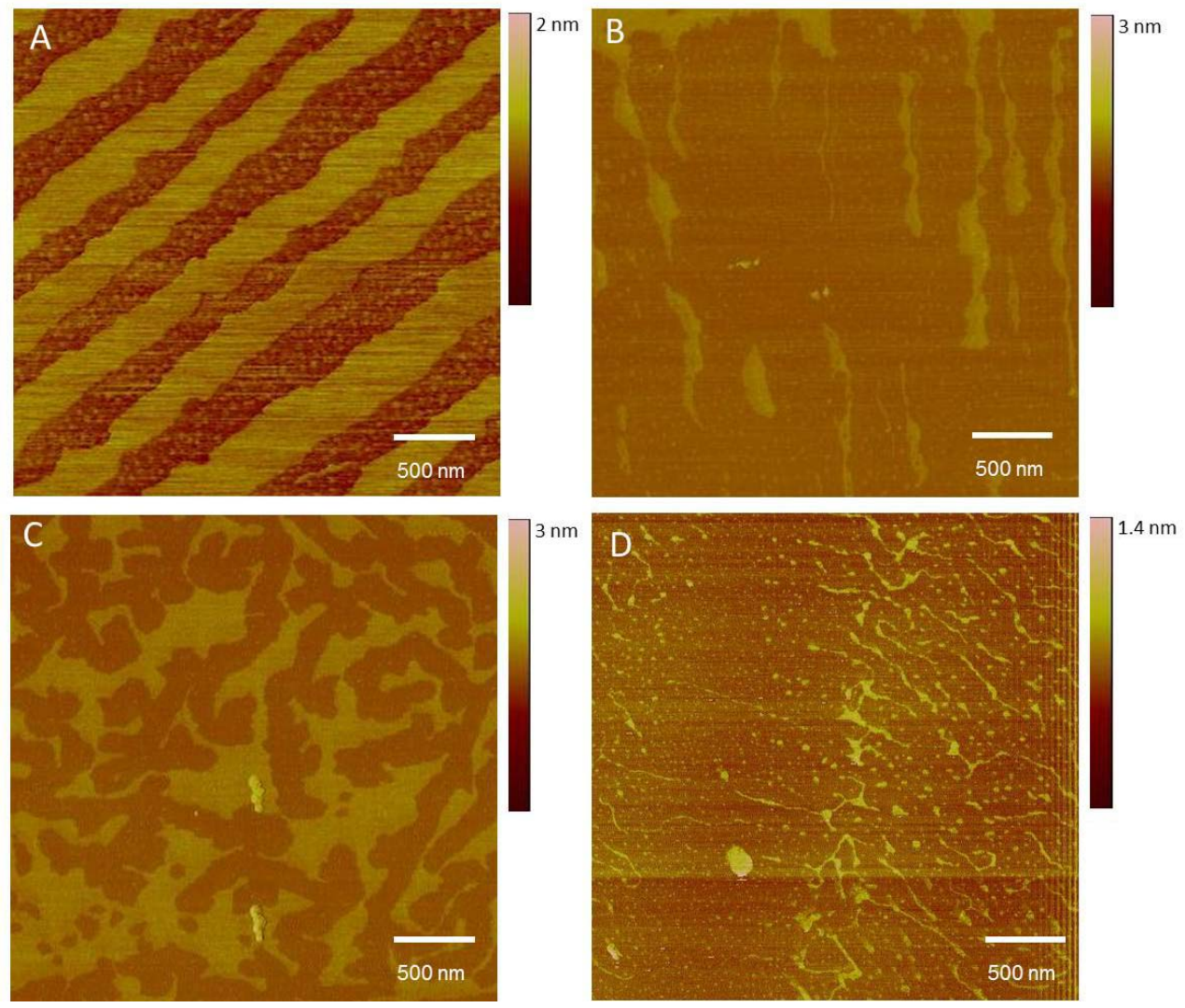

Figure 4. AFM height analysis of DPPC lipid monolayers $(12 \mathrm{mN} / \mathrm{m})$ in the presence of different concentrations (v/v) of G4 biotinylated PAMAM dendrimers: (A) DPPC:G4bio, 2:1; (B) DPPC:G4bio, 1:1; (C) DPPC:G4bio, 1:1; (D) DPPC:G4bio, 1:2.

viable cells (Figure 5). LDH is a soluble cytosolic enzyme that is released into the culture medium following loss of membrane integrity resulting from either apoptosis or necrosis. LDH activity is commonly used as an indicator of cell membrane integrity and serves as a general means to assess cytotoxicity resulting from chemical compounds or environmental toxic factors. Briefly, LDH reduces $\mathrm{NAD}+$, which then converts a tetrazolium dye to a soluble, colored formazan derivative. The absorbance of the converted dye is measured at a wavelength of 490 $\mathrm{nm}$ [40-42]. Therefore, the higher the absorbance, the more cell damage. While certain nanoparticles have been shown to inactivate $\mathrm{LDH}$, producing false negative toxicity results [43], this is not a concern with the dendrimers in the study.

As shown in Figure 5, bEnd.3 cells were exposed to one of the following treatment conditions: G4 biotinylated PAMAM dendrimers; G4 PAMAM dendrimers; culture media alone (negative control); or sodium azide (positive control). After $24 \mathrm{~h}$ no significant differences were detected in the amount of $\mathrm{LDH}$ produced by the cells across the four treatment groups $[\mathrm{F}(3,11)=2.892, \mathrm{p}$ $>0.05$ ]. However, after $48 \mathrm{~h}$, cells produced significantly more LDH $[F(3,11)=40.015, \mathrm{p}<0.001]$. Post-hoc analyses show that both types of dendrimers and sodium azide were more toxic than media alone. Additionally, biotinylated dendrimers were more toxic to the cells than any of the other treatment groups. These findings suggest that biotinylation of G4 PAMAM dendrimers results in higher levels of toxicity than non-biotinylation in this cell culture system. Cell toxicity measurements are in

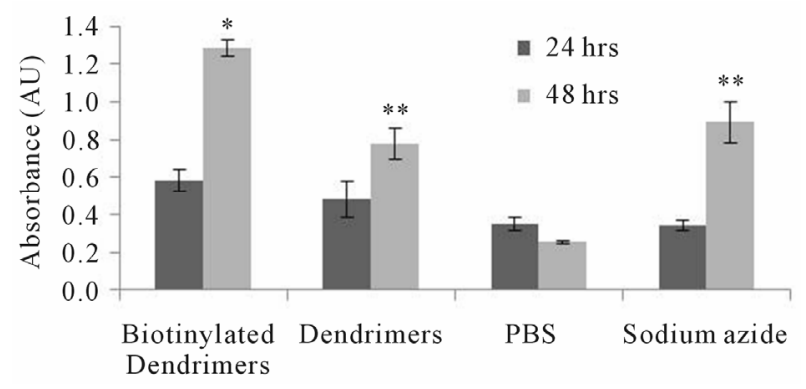

Figure 5. LDH activity of bEND.3 cells exposed to G4 biotinylated PAMAM dendrimers, G4 PAMAM dendrimers, or sodium azide. Values represent mean \pm SEM. *p < 0.001 relative to media alone, dendrimers and sodium azide; ** $\mathbf{p}<0.001$ relative to media alone. 
agreement with Langmuir compression isotherms and AFM analyses. Although it is important to note that the dosage of dendrimers may impact toxicity measurements [44].

\section{Conclusions}

The findings presented here show the potential toxicity of G4 and G4 biotinylated PAMAM dendrimers and how biophysical measurements with model lipid systems can be correlated with cell toxicity analysis to provide information on nanoparticle toxicity. At this time the exact factors that lead to the increased toxicity measured for biotinylated PAMAM dendrimers are unknown. However, controlling the degree of surface functionalization could potentially reduce dendrimer toxicity [22] (currently $\sim 17 \%$ surface coverage) [30]. It is possible that biotinylated PAMAM dendrimers may prove to be more toxic compared to PAMAM dendrimers alone, in part, due to their potential mechanism of uptake across the BBB, as biotin has shown to cross the BBB through carrier mediated endocytosis [24,25]. Therefore, there may be more biotinylated PAMAM dendrimer conjugates getting into cells compared to non-biotinylated PAMAM dendrimers, leading to more cell death. Although it is important to note that the dosage of dendrimers may impact toxicity measurements [44].

\section{Acknowledgements}

This project has been funded by the Merck Institute for Science Education, Kentucky NSF EPSCoR, Northern Kentucky University, Center for Integrated Natural Science and Mathematics and the NKU Research Foundation. This work was also supported by a grant from the Kentucky Biomedical Research Infrastructure Network (P20 RR016481-08) and the National Science Foundation (DMR-0526686, CHE-0619342).

\section{REFERENCES}

[1] R. Dermietzel and D. Krause, "Molecular Anatomy of the Blood-Brain Barrier as Defined by Immunocytochemistry," International Review Cytology, Vol. 127, No. 1991, pp. 57-109.

[2] T. S. Reese and M. J. Karnovsky, "Fine Structural Localization of a Blood-Brain Barrier to Exogenous Peroxidase," The Journal of Cell Biology, Vol. 34, No. 1, 1967, pp. 207-217. doi:10.1083/jcb.34.1.207

[3] G. Savettieri, I. Di Liegro, C. Catania, L. Licata, G. L. Pitarresi, S. D’Agostino, G. Schiera, V. De Caro, G. Giandalia, L. I. Giannola and A. Cestelli, "Neurons and ECM Regulate Occludin Localization in Brain Endothelial Cells," NeuroReport, Vol. 11, No. 5, 2000, pp. 1081-1084. doi:10.1097/00001756-200004070-00035

[4] K. Mertsch and M. Jochen, "Blood-Brain Barrier Penetra- tion and Drug Development from an Industrial Point of View," Current Medicinal Chemistry: Central Nervous System Agents, Vol. 2, No. 3, 2002, pp. 187-201. doi:10.2174/1568015023358067

[5] H. Yang, "Nanoparticle-Mediated Brain-Specific Drug Delivery, Imaging and Diagnosis," Pharmaceutical Research, Vol. 27, No. 9, 2010, pp. 1759-1771. doi:10.1007/s11095-010-0141-7

[6] S. Bhaskar, F. Tian, T. Stoeger, W. Kreyling, J. de la Fuente, V. Grazú, P. Borm, G. Estrada, V. Ntziachristos and D. Razansky, "Multifunctional Nanocarriers for Diagnostics, Drug Delivery and Targeted Treatment across Blood-Brain Barrier: Perspectives on Tracking and Neuroimaging," Particle and Fibre Toxicology, Vol. 7, No. 3, 2010, pp. 1-25.

[7] A. Agarwal, N. Lariya, G. Saraogi, N. Dubey, H. Agrawal and G. P. Agrawal, "Nanoparticles as Novel Carrier for Brain Delivery: A Review," Current Pharmaceutical Design, Vol. 15, No. 8, 2009, pp. 917-925. doi: $10.2174 / 138161209787582057$

[8] J. L. Gilmore, Y. Xiang, Q. Lingdong and A. Kabanov, "Novel Nanomaterials for Clinical Neuroscience," Journal of NeuroImmune Pharmacology, Vol. 3, No. 2, 2008, pp. 83-94. doi:10.1007/s11481-007-9099-6

[9] G. M. Dykes, "Dendrimers: A Review of Their Appeal and Applications," Journal of Chemical Technology and Biotechnology, Vol. 76, No. 9, 2001, pp. 903-918. doi:10.1002/jctb.464

[10] D. A. Tomalia, "Birth of a New Macromolecular Architecture: Dendrimers as Quantized Building Blocks for Nanoscale Synthetic Organic Chemistry," Progress in Polymer Science, Vol. 30, No. 3-4, 2005, pp. 294-324. doi:10.1016/j.progpolymsci.2005.01.007

[11] D. A. Tomalia, "The Emergence of a New Macromolecular Architecture: The Dendritic State,” In: J. E. Mark, Ed., Physical Properties of Polymers Handbook, Springer, New York, 2007, pp. 671-692. doi:10.1007/978-0-387-69002-5 42

[12] D. A. Tomalia, S. A. Henderson and M. S. Diallo, "Dendrimers-An Enabling Synthetic Science to Controlled Organic Nanostructures," In: W. A. I. Goddard, D. W. Brenner, S. E. Lyshevski and G. J. Iafrate, Eds., Handbook of Nanoscience, Engineering and Technology, CRC Press, Boca Raton, 2007, pp. 24.1-24.47. doi:10.1201/9781420007848.ch24

[13] D. Astruc, E. Boisselier and C. Ornelas, "Dendrimers Designed for Functions: From Physical, Photophysical and Supramolecular Properties to Applications in Sensing, Catalysis, Molecular Electronics, Photonics and Nanomedicine," Chemical Reviews, Vol. 110, No. 4, 2010, pp. 1857-1959.doi:10.1021/cr900327d

[14] V. Gajbhiye, V. K. Palanirajan, R. K. Tekade and N. K. Jain, "Dendrimers as Therapeutic Agents: A Systematic Review," Journal of Pharmacy and Pharmacology, Vol. 61, No. 8, 2010, pp. 989-1003. doi:10.1211/jpp.61.08.0002

[15] M. A. Mintzer and M. W. Grinstaff, "Biomedical Appli- 
cations of Dendrimers: A Tutorial," Chemical Society Reviews, Vol. 40, No. 1, 2011, pp. 173-190. doi:10.1039/b901839p

[16] K. C. Petkar, S. S. Chavhan, S. Agatonovik-Kustrin and K. K. Sawant, "Nanostructured Materials in Drug and Gene Delivery: A Review of the State of the Art," Critical Reviews in Therapeutic Drug Carrier Systems, Vol. 28, No. 2, 2011, pp. 101-164.

[17] W. Wijagkanalan, S. Kawakami and M. Hashida, "Designing Dendrimers for Drug Delivery and Imaging: Pharmacokinetic Considerations," Pharmaceutical Research, Vol. 28, No. 7, 2011, pp. 1500-1019. doi:10.1007/s11095-010-0339-8

[18] U. Boas and P. M. Heegaard, "Dendrimers in Drug Research," Chemical Society Reviews, Vol. 33, No. 1, 2004, pp. 43-63. doi:10.1039/b309043b

[19] R. Huang, W. Ke, L. Han, Y. Liu, K. Shao, L. Ye, J. Lou, C. Jiang and Y. Pei, "Brain-Targeting Mechanisms of Lactoferrin-Modified DNA-Loaded Nanoparticles," Journal Cerebral Blood Flow \& Metabolism, Vol. 29, 2009, pp. 1914-1923.

[20] G. Wu, R. F. Barth, W. Yang, S. Kawabata, L. Zhang and K. Green-Church, "Targeted Delivery of Methotrexate to Epidermal Growth Factor Receptor-Positive Brain Tumors by Means of Cetuximab (IMC-C225 Dendrimer Bioconjugates," Molecular Cancer Therapeutics, Vol. 5, 2006, pp. 52-59.

[21] T. L. Kaneshiro and Z. R. Lu, "Targeted Intracellular Codelivery of Chemotherapeutics and Nucleic Acid with a Well-Defined Dendrimer-Based Nanoglobular Carrier," Biomaterials, Vol. 30, 2009, pp. 5660-5666.

[22] R. Duncan and L. Izzo, "Dendrimer Biocompatibility and Toxicity," Advanced Drug Delivery Reviews, Vol. 57, No. 15, 2005, pp. 2215-2237. doi:10.1016/j.addr.2005.09.019

[23] K. M. Kitchens, M. E. El-Sayed and H. Ghandehari, "Transepithelial and Endothelial Transport of Poly(amidoamine) Dendrimers," Advanced Drug Delivery Reviews, Vol. 57, No. 2005, pp. 2163-2176.

[24] R. Spector and D. Mock, "Biotin Transport through the Blood-Brain Barrier," Journal of Neurochemistry, Vol. 48, No. 2, 1987, pp. 400-404. doi:10.1111/j.1471-4159.1987.tb04107.x

[25] F. Shi, C. Bailey, A. W. Malick and K. L. Audus, "Biotin Uptake and Transport across Bovine Brain Microvessel Endothelial Cell Monolayers," Pharmaceutical Research, Vol. 10, 1993, pp. 282-288.

[26] N. Sato, H. Kobayashi, T. Saga, Y. Nakamoto, T. Ishimori, K. Togashi, Y. Fujibayashi, J. Konishi and M. W. Brechbiel, "Tumor Targeting and Imaging of Interaperitoneal Tumors by Use of Antisense Oligo-DNA Complexed with Dendrimers and/or Avidin in Mice," Clinical Cancer Research, Vol. 7, 2001, pp. 3606-3612.

[27] D. S. Wilbur, P. M. Pathare, D. K. Hamlin, K. R. Buhler and R. L. Vessella, "Biotin Reagents for Antibody Pretargeting. 3. Synthesis, Radioiodination and Evaluation of
Biotinylated Starburst Dendrimers," Bioconjugate Chemistry, Vol. 9, No. 6, 1998, pp. 813-825. doi: $10.1021 / \mathrm{bc} 980055 \mathrm{e}$

[28] H. C. Yoon, M. Y. Hong and H. S. Kim, "Affinity Biosensor for Avidin Using a Double Functionalized Dendrimer Monolayer on a Gold Electrode," Analytical Biochemistry, Vol. 282, No. 1, 2000, pp. 121-128. doi:10.1006/abio.2000.4608

[29] S. Beg, A. Samad, M. I. Alam and I. Nazish, "Dendrimers as Novel Systems for Delivery of Neuropharmaceuticals to the Brain," CNS \& Neurological Disorders-Drug Targets, Vol. 10, No. 5, 2011, pp. 576-588.

[30] C. A. Cason, S. A. Oehrle, T. A. Fabre, C. Girten, K. A. Walters, D. A. Tomalia, K. L. Haik and H. A. Bullen, "Improved Methodology for Monitoring Poly (amidoamine) Dendrimers Surface Transformations and Product Quality by Ultra Performance Liquid Chromatography," Journal of Nanomaterials, Vol. 2008, 2008, Article ID 456082, 7 Pages.

[31] R. Montesano, M. S. Pepper, U. Mohle-Steinlein, W. Risau, W. F. Wagner and L. Orci, "Increased Proteolytic Activity Is Responsible for the Aberrant Morphogenetic Behavior of Endothelial Cells Expressing the Middle T Oncogene," Cell, Vol. 62, No. 3, 1990, pp. 435-445. doi:10.1016/0092-8674(90)90009-4

[32] K. S. Birdi, "Dipalmitoyllecithin Monolayers at the Air/ Water Interface," Langmuir, Vol. 3, No. 1987, pp. 132133.

[33] K. J. Klopfer and T. K. Vanderlick, "Isotherms of Dipalmitoylphosphatidylcholine (DPPC) Monolayers: Features Revealed and Features Obscured," Journal of Colloid and Interface Science, Vol. 182, No. 1, 1996, pp. 220-229. doi:10.1006/jcis.1996.0454

[34] C. Peetla and V. Labhasetwar, "Effect of Molecular Structure of Cationic Surfactants on Biophysical Interactions of Surfactant-Modified Nanoparticles with a Model Membrane and Cellular Uptake," Langmuir, Vol. 25, No. 4, 2009, pp. 2369-2377. doi:10.1021/la803361y

[35] C. Nunes, G. Brezesinski, C. Pereira-Leite, J. L. Lima, S. Reis and M. Lúcio, "NSAIDs Interactions with Membranes: A Biophysical Approach," Langmuir, Vol. 27, No. 17, 2011, pp. 10847-10858. doi:10.1021/la201600y

[36] R. K. Harishchandra, M. Saleem and H.-J. Galla, "Nanoparticle Interaction with Model Lung Surfactant Monolayers," Journal of the Royal Society Interface, Vol. 7, Suppl. 1, 2010, pp. S15-S26.

[37] V. Tiriveedhi, K. M. Kitchens, K. J. Nevels, H. Ghandehari and P. Butko, "Kinetic Analysis of the Interaction between Poly(amidoamine) Dendrimers and Model Lipid Membranes," Biochimica et Biophysica Acta, Vol. 1808 , No. 1, 2011, pp. 209-218. doi:10.1016/j.bbamem.2010.08.017

[38] H. Bensikaddour, N. Fa, I. Burton, M. Deleu, L. Lins, A. Schanck, R. Brasseur, Y. F. Dufrêne, E. Goormaghtigh and M. P. Mingeot-Leclercq, "Characterization of the Interactions between Fluoroquinolone Antibiotics and Lip- 
ids: A Multitechnique Approach," Biophysical Journal, Vol. 94, No. 8, 2008, pp. 3035-3046. doi:10.1529/biophysj.107.114843

[39] A. Mecke, S. Uppuluri, T. M. Sassanella, D.-K. Lee, A. Ramamoorthy, J. R. J. Baker, B. G. Orr and M. M. Banaszak Holl, "Direct Observation of Lipid Bilayer Disruption by Poly(amidoamine) Dendrimers," Chemistry and Physics of Lipids, Vol. 132, No. 2004, pp. 3-14.

[40] E. Bonfoco, D. Krainc, M. Ankarcrona, P. Nicotera and S. A. Lipton, "Apoptosis and Necrosis: Two Distinct Events Induced, Respectively, by Mild and Intense Insults with $\mathrm{N}$-methyl-D-aspartate or Nitric Oxide/Superoxide in Cortical Cell Cultures," Proceedings of the National Academy of Sciences, Vol. 92, No. 16, 1995, pp. 7162-7166. doi:10.1073/pnas.92.16.7162

[41] T. Decker and M.-L. Lohmann-Matthes, "A Quick and Simple Method for the Quantitation of Lactate Dehydrogenase Release in Measurements of Cellular Cytotoxicity and Tumor Necrosis Factor (TNF) Activity," Journal of
Immunological Methods, Vol. 15, No. 1998, pp. 61-69.

[42] C. Legrand, J. Bour, C. Jacob, J. Capiaumont, A. Martial, A. Marc, M. Wudtke, G. Kretzmer, C. Demangel and D. Duval, "Lactate Dehydrogenase (LDH) Activity of the Cultured Eukaryotic Cells as Marker of the Number of Dead Cells in the Medium," Journal of Biotechnology, Vol. 25, No. 1995, pp. 231-243.

[43] X. Han, R. Gelein, N. Corson, P. Wade-Mercer, J. Jiang, P. Biswas, J. N. Finkelstein, A. Elder and G. Oberdorster, "Validation of an LDH Assay for Assessing Nanoparticle Toxicity," Toxicology, Vol. 287, No. 1-3, 2011, pp. 99104. doi:10.1016/j.tox.2011.06.011

[44] J. Teeguarden, P. Hinderliter, G. Orr, B. Thrall and J. Pounds, "Particokinetics in Vitro: Dosimetry Considerations for in Vitro Nanoparticle Toxicity Assessments," Toxicological Sciences: An Official Journal of the Society of Toxicology, Vol. 95, No. 2, 2007, pp. 300-312. doi:10.1093/toxsci/kfl165 